\title{
Bargmann's Inequalities in Spaces of Arbitrary Dimension
}

By

\author{
Noriaki SETô*
}

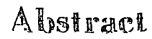

An inequality is derived which gives an upper bound of the number of bound states in the $l$-th partial wave $(l=0,1, \cdots)$ of the two-body Schrödinger equation with a spherically symmetric potential function in the $n$-dimensional space $(n=1,2 \cdots)$. This is a generalization of Bargmann's inequality for the case $n=3$. The generalization is straightforward for the case $l \geqq 1$ with $n=2$ and $l \geqq 0$ with $n \geqq 3$. After a mathematically rigorous justification of his heuristic argument, Schwinger's method in his simple proof of Bargmann's inequality is employed here. Newton's result for the case $l=-\frac{1}{2}, n=3$, which is equivalent to the case $l=0, n=2$, is reobtained.
\end{abstract}

\section{$\S$ 耳. Introduction}

In the nonrelativistic quantum mechanics, Bargmann [1] has obtained in 1952 the so-called "Bargmann's inequality" which gives an upper bound for the number of bound states produced, in the two-body system, by a spherically symmetric potential. His method of proof is to count the number of zeros of the zero-energy solution, with a smoothness condition at the origin, of the partial-wave Schrödinger equation and to make use of the well-known correspondence between the number of zeros and that of bound states. Avoiding this highly complicated method, Schwinger $\lceil 2\rceil$ has elegantly rederived, in a heuristic manner, Bargmann's inequality and its versions, by transforming the zero-energy "hound state" problem to an integral equation and applying Mercer's theorem to the positive integral operator thus obtained. Soon after, an inequality for the case $l=-\frac{1}{2}$,

Communicated by S. Matsuura, February 2, 1973.

* Department of Applied Mathematics and Physics, Faculty of Engineering, Kyoto Inivessity, Kyoto. 
important in the theory of complex angular momenta, was obtained by Newton [3] by employing a perturbation method which becomes exact in the $\operatorname{limit} l \rightarrow-\frac{1}{2}$.

Above arguments, however, are restricted to our real space, that is, to the three-dimensional space. In the case of one- and two-dimensional spaces, as is well known in exercises on quantum mechanics, an attractive square-well potential with a fixed radius can produce at least one bound state, however shallow the well may be. This suggests that, in the oneand two-dimensional cases, an inequality for the number of bound states will take a form drastically different from that in the three-dimensional space.

The purpose of the present paper is to generalize Bargmann's inequality to the space of arbitrary dimension and to justify in a mathematically rigorous way the heuristic arguments employed thus far.

General formalism is given in the next section for two- or higherdimensional case. A straightfoward extension of Bargmann's inequality to the two- or higher-dimensional space except for the zero-th wave in the two-dimensional space is performed in Sec. 3. Heuristic derivations are given for the one-dimensional case in Sec. 4 and for the case $l=0$ in the two-dimensional case in Sec. 5. Section 6 is devoted to the justification of arguments in the preceding two sections. In the final section are given a summary and discussions.

\section{§ 2. General Formalism}

In polar coordinates, the $n$-dimensional $(n \geqq 2)$ Laplacian $\Delta_{n}$ is expressed as

$$
\Delta_{n}=\frac{1}{r^{n-1}} \frac{\partial}{\partial r}\left(r^{n-1} \frac{\partial}{\partial r}\right)+\frac{1}{r^{2}} \mathbb{A}_{n}^{2}
$$

where $r$ is the radial variable and $\Lambda_{n}^{2}$ is the Laplacian on the $(n-1)$ dimensional unit sphere. The Laplacian $\Lambda_{n}^{2}$ has a series of eigenvalues $-l(l+n-2)(l=0,1, \ldots)$ each of which has a degree of degeneracy $[4]$

$$
h(n, l)=\frac{(2 l+n-2) \Gamma(n+l-2)}{\Gamma(n-1) \Gamma(l+1)} .
$$


Here $h(2,0)$ must be interpreted as a limit $\lim _{n \rightarrow 2} h(n, 0)=1$.

By the separation of variables for the $n$-dimensional Schrödinger equation with a spherically symmetric potential $V=V(r)$ :

$$
\left[-\Delta_{n}+V(r)\right] \psi_{n}(\dot{x})=E \psi_{n}(\vec{x}), \vec{x} \in R^{n} \quad(E \text { : energy })
$$

into the radial part and angular part, the radial part in the $l$-th wave takes the form

$$
\left[-\frac{1}{r^{n-1}} \frac{d}{d r}\left(r^{n-1} \frac{d}{d r}\right)+\frac{l(l+n-2)}{r^{2}}+V(r)\right] \psi_{n}^{l}(r)=E \psi_{n}^{l}(r)
$$

In terms of a function $\phi_{n}^{l}$ defined by

$$
\phi_{n}^{l}(r)=r^{(n-1) / 2} \psi_{n}^{l}(r),
$$

Eq. (2.2) is expressed as

$$
\left[-\left(\frac{d}{d r}\right)^{2}+\frac{(l+(n-1) / 2)(l+(n-3) / 2)}{r^{2}}+V(r)\right] \phi_{n}^{l}(r)=E \phi_{n}^{l}(r) .
$$

As was shown by Kodaira [5], the eigenvalue problem (2.4) is, in the Hilbert space $L^{2}((0, \infty) ; d r)$, well defined for the case $2 l+n-4 \geqq 0$, provided that the potential function is a real-valued piecewise-continuous function satisfying the condition

$$
\int_{0}^{\infty} r|V(r)| d r<\infty
$$

For the case $n=3, l=0$, in order to make the eigenvalue problem well defined, we must require a boundary condition $\phi_{3}^{0}(r)=O(r)$ as $r \rightarrow 0$ for the eigenfunctions in addition to the same conditions on the potential as above. As for the case $n=2, l=0$, we shall impose a stronger condition on the potential;

$$
\int_{0}^{\infty} r(|\log r|+1)|V(r)| d r<\infty
$$

and require a boundary condition $\phi_{2}^{0}(r)=O\left(r^{1 / 2}\right)$ as $r \rightarrow 0$.

It must be noticed that the behaviour of the potential as $r \rightarrow \infty$ is more restrictive than that given in Ref. [5], to guarantee the finiteness of the 
number of point eigenvalues. It was also shown [5] that each point eigenvalue $E$ (the energy level of the bound state), if any, is non-positive. Hereafter, we shall set $E=-k^{2}$ and for the point eigenvalues we shall take the branch $k \geqq 0$.

First of all, we shall summarize the well-known properties of the solution of Eq. (2.4) in the following lemma (for the proof see, for instance, Ref. [6]):

\section{Lemma 2.1.}

a) The case $2 l+n-3 \geqq 0$

Under the condition (2.5), any non-trivial solution $\phi_{n}^{l}$ of Eq. (2.4) behaves, for any $k$, near the origin such that $\lim r^{l+(n-3) / 2} \cdot \phi_{n}^{l}(r)$ exists, and if the limit vanishes, then $\lim r^{-l-(n-1) / 2} \phi_{n}^{l}(r)$ exists and does not vanish. For $k>0, e^{-k r} \circ \phi_{n}^{l}(r)$ tends to a finite limit as $r \rightarrow \infty$, and if the limit vanishes, then $e^{k r} \cdot \phi_{n}^{l}(r)$ tends to a non-zero finite limit. For $k=0, r^{-l-(n-1) / 2} \phi_{n}^{l}(r)$ tends to a finite limit as $r \rightarrow \infty$, and if the limit vanishes, then $r^{l+(n-3) / 2} \phi_{n}^{l}(r)$ tends to a non-zero finite limit.

b) The case $n=2, l=0$

Under the condition (2.6), any non-trivial solution $\phi_{2}^{0}$ of Eq. (2.4) behaves, for any $k$, near the origin such that $\lim _{r \rightarrow 0} r^{-1 / 2}|\log r|^{-1} \circ \phi_{2}^{0}(r)$ exists, and if the limit vanishes, then $\lim _{r \rightarrow 0} r^{-1 / 2} \phi_{2}^{0}(r)$ exists and does not vanish. For $k>0, e^{-k r}{ }_{\circ} \phi_{2}^{0}(r)$ tends to $a$ finite limit as $r \rightarrow \infty$, and if the limit vanishes, then $e^{k r} \cdot \phi_{2}^{0}(r)$ tends to a non-zero finite limit. For $k=0$, $r^{-1 / 2}|\log r|^{-1} \cdot \phi_{2}^{0}(r)$ tends to a finite limit as $r \rightarrow \infty$, and if the limit vanishes, then $r^{-1 / 2} \cdot \phi_{2}^{0}(r)$ tends to a non-zero finite limit.

As is easily seen from the above lemma, the wave function $\phi_{n}^{\prime}$ of a bound state with negative energy level $(k>0)$ behaves as

$$
\phi_{n}^{\prime}(r)= \begin{cases}O\left(r^{l+(n-1) / 2}\right), & r \rightarrow 0, \\ O\left(e^{-k r}\right), & r \rightarrow \infty .\end{cases}
$$

Therefore such a function $\phi_{n}^{l}$ belongs to $\mathscr{H}_{V} \equiv L^{2}((0, \infty) ;|V(r)| d r)$, as well as to $L^{2}((0, \infty) ; d r)$.

According to Bargmann's idea [1], we first replace the potential $V$ by a "more attractive" potential $-|V|$ or $V_{-}=\min [V, 0]$. 
Lemma 2.2. The number of point eigenvalues never decreases by the above replacement.

Proof: Consider a bound state problem

$$
\begin{array}{r}
{\left[-\left(\frac{d}{d r}\right)^{2}+\frac{(l+(n-1) / 2)(l+(l-3) / 2)}{r^{2}}+(1-\varepsilon) V(r)+\varepsilon(-|V(r)|)\right] \phi_{n}^{l}(r)} \\
=-k^{2} \phi_{n}^{l}(r)
\end{array}
$$

depending on a parameter $\varepsilon(0 \leqq \varepsilon \leqq 1)$. As is easily verified, each eigenvalue is non-degenerate for fixed $\varepsilon$. Using a standard technic of perturbation theory [7], we can show that, for each eigenvalue $-k_{i}^{2}$ and a corresponding eigenfunction $\phi_{i}$, the equality

$$
2 k_{i} \cdot \frac{d k_{i}}{d \varepsilon}=\left.\int_{0}^{\infty}(|V(r)|+V(r)) i \phi_{i}(r)\right|^{2} d r / \int_{0}^{\infty}\left|\phi_{i}(r)\right|^{2} d r
$$

holds. This implies $k_{i}$ is a non-decreasing function of $\varepsilon$. Therefore the number of bound states generated by the potential $V$, i.e. at $\varepsilon=0$, never decreases by the above replacement $\varepsilon=1$. (The proof of this lemma and all the subsequent arguments go parallel for the replacement of $V$ by $V_{-}$.)

Next, following Schwinger's method [2], we shall replace the potential $-|V|$ by a potential $-\lambda|V|$ and consider a bound state problem

$$
\begin{array}{r}
{\left[-\left(\frac{d}{d r}\right)^{2}+\frac{(l+(n-1) / 2)(l+(n-3) / 2)}{r^{2}}-\lambda|V(r)|\right] \phi_{n}^{l}(r)} \\
=-k^{2} \phi_{n}^{l}(r) .
\end{array}
$$

It can be shown that, for fixed positive $\lambda$, all the eigenvalues $-k_{i}(\lambda)^{2}$, if any, are non-degenerate and each $k_{i}(\lambda)$ satisfies

$$
2 k_{i}(\lambda) \frac{d k_{i}(\lambda)}{d \lambda}=\int_{0}^{\infty}\left|\phi_{l l}^{l}(r ; i)\right|^{2}|V(r)| d r / \int_{0}^{\infty}\left|\phi_{n}^{l}(r ; i)\right|^{2} d r
$$

where $\phi_{n}^{l}(\cdot ; i)$ is a corresponding eigenfunction. Consequently $k_{i}(\lambda)$ decreases monotonically as $\lambda$ decreases so long as $k_{\imath}(\lambda)$ remains positive. Since all bound states disappear at $\lambda=0$, there exists a point $\lambda_{i}$ on the interval $[0,1)$, such that $k_{i}\left(\lambda_{i}\right)=0$. Thus Schwinger has reduced the eigenvalue problem for $-k^{2}$ with $\lambda=1$ in Eq. (2.8) to the eigenvalue 
problem for $\lambda \in[0,1)$ with $k=0$ in Eq. (2.8). There exists, however, a subtle point in taking the limit $k \rightarrow 0$ in Eq. (2.8), since as is seen in Lemma 2.1, the asymptotic behaviour of the solution of Eq. (2.8) changes drastically for $k>0$ and for $k=0$.

In the next section, we shall justify Schwinger's argument and obtain a generalization of Bargmann's inequality for the case $2 l+n-2 \geqq 1$.

\section{§3。 Higheromimensional Case}

$$
-2 l+2 \geqq \mathbb{1}-
$$

We shall try, in this section, to derive a generalized Bargmann's inequality for the case $2 l+n-2 \geqq 1$, that is, for all $l$ with $n \geqq 3$ and $l \geqq 1$ with $n=2$.

Hereafter we shall mainly confine ourselves to the Hilbert space $\mathscr{H}_{V}=L^{2}((0, \infty) ;|V(r)| d r)$, and hence, for notational convenience, we shall write $d \mu(r)$ instead of $|V(r)| d r$ and denote by $(),\|\|,,\|\|_{2}$ and $\operatorname{tr}(\mathrm{)}$ the inner product, the norm, the Hilbert-Schmidt norm and the trace on $\mathscr{H}_{V}$, respectively.

First we shall rewrite the eigenvalue equation (2.8) into an integral equation:

Lemma 3.1. The bound state problem (2.8) is, for $k>0$, equivalent to the integral equation

$$
\phi_{n}^{l}=\lambda G_{n}^{l}(k) \phi_{n}^{l}
$$

on $\mathscr{H}_{V}$, where the kernel function of the integral operator $G_{n}^{l}(k)$ is given by

$$
G_{n}^{l}(k)\left(r, r^{\prime}\right)=\left(r r^{\prime}\right)^{1 / 2} \times \begin{cases}I_{l+\frac{1}{2} n-1}(k r) \cdot K_{l+\frac{1}{2} n-1}\left(k r^{\prime}\right), & 0 \leqq r<r^{\prime} \\ I_{l+\frac{1}{2} n-1}\left(k r^{\prime}\right) \cdot K_{l+\frac{1}{2} n-1}(k r), & r>r^{\prime}\end{cases}
$$

Proof: Using the properties of modified Bessel functions, $I_{\nu}$ and $K_{\nu}$, we can easily recognize that the solution of Eq. (3.1) satisfies Eq. (2.8). Therefore we have only to show that the solution satisfies the boundary condition (2.7). Since the explicit form of Eq. (3.1) is 


$$
\phi_{n}^{l}(r)=\lambda \int_{0}^{\infty} G_{n}^{l}(k)\left(r, r^{\prime}\right) \phi_{n}^{l}\left(r^{\prime}\right) d \mu\left(r^{\prime}\right)
$$

the estimates on $\phi_{n}^{l}$ can be obtained through the use of Schwarz's inequality as

$$
\begin{aligned}
\left|\phi_{n}^{l}(r)\right| & \leqq|\lambda|\left\{\int_{0}^{\infty}\left|G_{n}^{l}(k)\left(r, r^{\prime}\right)\right|^{2} d \mu\left(r^{\prime}\right)\right\}^{1 / 2}\left\{\int_{0}^{\infty}\left|\phi_{n}^{l}\left(r^{\prime}\right)\right|^{2} d \mu\left(r^{\prime}\right)\right\}^{1 / 2} \\
& \equiv|\lambda|\left\|G_{n}^{l}(k)(r, \circ)\right\| \cdot\left\|\phi_{n}^{l}\right\| .
\end{aligned}
$$

The second factor on the right hand side of this inequality is estimated as

$$
\begin{aligned}
\left\|G_{n}^{l}(k)(r, \cdot)\right\|^{2}=r\left(K_{\nu}(k r)\right)^{2} \cdot \int_{0}^{r} r^{\prime}\left(I_{\nu}\left(k r^{\prime}\right)\right)^{2} d \mu\left(r^{\prime}\right) \\
\quad+r\left(I_{\nu}(k r)\right)^{2} \cdot \int_{r}^{\infty} r^{\prime}\left(K_{\nu}\left(k r^{\prime}\right)\right)^{2} d \mu\left(r^{\prime}\right) \\
\quad \leqq r\left(I_{\nu}(k r) \cdot K_{\nu}(k r)\right)^{2} \int_{0}^{\infty} r^{\prime} d \mu\left(r^{\prime}\right), \quad\left(\nu \equiv l+\frac{1}{2} n-1 \geqq \frac{1}{2}\right)
\end{aligned}
$$

where we have made use of the property that $I_{\nu}\left(K_{\nu}\right)$ is a positive-valued monotonously increasing (decreasing) function. As is easily verified, the function $I_{\nu}(x) K_{\nu}(x)$, for $\nu \geqq \frac{1}{2}$, is a bounded function for $x \geqq 0$. Consequently it follows

$$
\left|\phi_{n}^{l}(r)\right| \leqq|\lambda| C_{\nu}\left\|\phi_{n}^{l}\right\| r^{1 / 2} \quad\left(C_{\nu}: \text { positive constant }\right)
$$

From this estimate and Lemma 2.1, it is concluded that $\phi_{n}^{l}$ satisfies the boundary condition (2.7).

The converse statement, i.e., that the solution of Eq. (2.8) with the boundary condition (2.7) satisfies the integral equation (3.1) is trivially verified by the standard arguments.

As a byproduct, we can readily show through (3.3) that the operator $G_{n}^{l}(k)$ is of Hilbert-Schmidt type and its Hilbert-Schmidt norm

$$
\left\|G_{n}^{l}(k)\right\|_{2}=\left\{\int_{0}^{\infty} \int_{0}^{\infty}\left|G_{n}^{l}(k)\left(r, r^{\prime}\right)\right|^{2} d \mu\left(r^{\prime}\right) d \mu(r)\right\}^{1 / 2}
$$

is bounded by a constant independent of $k(>0)$. We can, however, show more about the properties of $G_{n}^{l}(k)$ : 
Lemma 3.2. The integral operator $G_{n}^{l}(k)$ is of positive type and belongs to the trace class, with its trace uniformly bounded with respect to $k(>0)$.

Proof: To prove the positivity, we have only to show that $\left(f, G_{n}^{l}(k) f\right)$ is non-negative for sufficiently well-behaved $f$, for instance, for infinitely differentiable function with compact support in $(0, \infty)$. For such a function, $\left(f, G_{n}^{l}(k) f\right)$ is equal to

$$
\int_{0}^{\infty}\left\{\left(-\left(\frac{d}{d r}\right)^{2}+\frac{\left(\nu-\frac{1}{2}\right)\left(\nu+\frac{1}{2}\right)}{r^{2}}+k^{2}\right) g(r)\right\}^{*} g(r) d r
$$

where $g=G_{n}^{l}(k) f$. This integral is evidently non-negative.

By the usual argument of the positive integral operator and by the fact that the kernel function of $G_{n}^{l}(k)$ is independent of the potential and it is a continuous function with respect to two variables, it immediately follows that

$$
a^{2} G_{n}^{l}(k)(r, r)-2 a G_{n}^{l}(k)\left(r, r^{\prime}\right)+G_{n}^{l}(k)\left(r^{\prime}, r^{\prime}\right) \geqq 0
$$

for any real $a$, which in turn implies

$$
G_{n}^{l}(k)\left(r, r^{\prime}\right)^{2} \leqq G_{n}^{l}(k)(r, r) \cdot G_{n}^{l}(k)\left(r^{\prime}, r^{\prime}\right) .
$$

Thus, by the integration of this inequality with respect to $d \mu(r) d \mu\left(r^{\prime}\right)$, we obtain

$$
\left\|G_{n}^{l}(k)\right\|_{2} \leqq \int_{0}^{\infty} G_{n}^{l}(k)(r, r) d \mu(r)
$$

Since the integral on the right hand side certainly exists and is bounded by a constant independent of $k(>0)$, we have again confirmed the property of $G_{n}^{l}(k)$ stated immediately after the proof of Lemma 3.1.

Denoting the eigenvalues of Eq. $(3.1)$ by $\lambda_{i}(k)(i=1,2, \ldots)\left(0<\lambda_{1}(k)<\right.$ $\left.\lambda_{2}(k)<\cdots\right)$, each of which is evidently non-degenerate, and corresponding orthonormal eigenfunctions by $\phi_{n}^{l}\left(\cdot, \lambda_{i}(k)\right)$, we get, from the positivity of $G_{n}^{l}(k)$, the inequality

$$
G_{n}^{l}(k)(r, r) \geqq \sum_{i=1}^{N} \frac{1}{\lambda_{i}(k)}\left|\phi_{n}^{l}\left(r, \lambda_{i}(k)\right)\right|^{2},
$$


for any positive integer $N$ and for $r$ belonging to the support of the measure $\mu$. Therefore

$$
\int_{0}^{\infty} G_{\|}^{l}(k)(r, r) d \mu(r) \geqq \sum_{i=1}^{\infty} \frac{1}{\lambda_{i}(k)} \equiv \operatorname{tr}\left(G_{n}^{l}(k)\right)
$$

In order to prove that the equality actually occurs in this inequality, it is sufficient to notice that, by a slight modification of the argument of Mercer's theorem [8], the right hand side of (3.5) converges uniformly on any compact subset of $[0, \infty)$ to the left-hand side as $N \rightarrow \infty$. Consequently we have

$$
\operatorname{tr}\left(G_{n}^{l}(k)\right)=\int_{0}^{\infty} r I_{l+\frac{1}{2} n-1}(k r) \cdot K_{l+\frac{1}{2} n-1}(k r)|V(r)| d r
$$

We shall next consider the zero-energy "bound state" problem, that is,

$$
\left[-\left(\frac{d}{d r}\right)^{2}+\frac{(l+(n-1) / 2)(l+(n-3) / 2)}{r^{2}}-\lambda|V(r)|\right] \phi_{n}^{l}(r)=0
$$

with the boundary condition

$$
\phi_{n}^{l}(r)= \begin{cases}O\left(r^{l+(n-1) / 2}\right), & r \rightarrow 0 \\ O\left(r^{-l-(n-3) / 2}\right), & r \rightarrow \infty\end{cases}
$$

In quite an analogous manner we can show the following

Lemma 3.3. The zero-energy "bound state" problem is equivalent to the integral equation

$$
\phi_{n}^{l}=\lambda G_{n}^{l} \phi_{n}^{l}
$$

on the Hilbert space $\mathscr{H}_{V}$, where the kernel function of the integral operator $G_{n}^{l}$ is given by

$$
G_{n}^{l}\left(r, r^{\prime}\right)=\frac{1}{2 l+n-2}\left(r r^{\prime}\right)^{1 / 2} \times \begin{cases}\left(r / r^{\prime}\right)^{l+(n-2) / 2}, & 0 \leqq r<r^{\prime} \\ \left(r^{\prime} / r\right)^{l+(n-2) / 2}, & r>r^{\prime}\end{cases}
$$

The integral operator $G_{n}^{l}$ is of positive type and belongs to the trace class with its trace given by 


$$
\operatorname{tr}\left(G_{n}^{l}\right)=\frac{1}{2 l+n-2} \int_{0}^{\infty} r|V(r)| d r
$$

Our main concern is how the Eq. (3.1) "approaches" Eq. (3.8) in the limit $k \rightarrow 0$.

Lemma 3.4. The operator $G_{n}^{l}(k)$ approaches $G_{n}^{l}$ as $k \rightarrow 0$ in the Hilbert-Schmidt norm, that is to say,

$$
\lim _{k \rightarrow 0}\left\|G_{n}^{l}(k)-G_{n}^{l}\right\|_{2}=0
$$

Proof: The proof is easily achieved by noting that the inequality $G_{n}^{l}(k)\left(r, r^{\prime}\right)^{2} \leqq C^{\prime} r r^{\prime}\left(C^{\prime}\right.$ : a constant independent of $k$ ) holds by virtue of (3.4) and that the kernel function (3.2) approaches pointwise to the kernel function (3.9) as $k \rightarrow 0$.

This lemma implies the following

Lemma 3.5. Let $\lambda_{i}(i=1,2, \ldots)\left(0<\lambda_{1}<\lambda_{2}<\cdots\right)$ be the eigenvalues of $G_{n}^{l}$. Then

$$
\lim _{k \rightarrow 0}\left|\frac{1}{\lambda_{i}(k)}-\frac{1}{\lambda_{i}}\right|=0 . \quad(i=1,2, \ldots)
$$

Proof: By means of a theorem due to Weyl and Courant [9], we have

$$
\left|\frac{1}{\lambda_{i}(k)}-\frac{1}{\lambda_{i}}\right| \leqq\left\|G_{n}^{l}(k)-G_{n}^{l}\right\|_{2} . \quad(i=1,2, \ldots)
$$

The proof follows from this inequality and Lemma 3.4.

We are now in a position to prove

Theorem 3.1. For $2 l+n-3>1$, the number of eigenvalues $\lambda_{i}$ of $G_{n}^{l}$ such that $0<\lambda_{i} \leqq 1$ is equal to the number of bound states generated by the potential $|V|$. For $1 \geqq 2 l+n-3 \geqq 0$, the number of eigenvalues $\lambda_{i}$ of $G_{n}^{l}$ such that $0<\lambda_{i}<1$ is equal to the number of bound states generated by the potential $|V|$.

Proof: Equation (2.9) shows that each eigenvalue $\lambda_{i}(k)$ is a monoto- 
nously-increasing differentiable function of $k$ for $k>0$. Moreover the fact that $\operatorname{tr}\left(G_{n}^{l}(k)\right)$ tends to zero as $k \rightarrow \infty$, which is readily verified through Eq. (3.6), implies that each $\lambda_{i}(k)$ tends to infinity as $k \rightarrow \infty$. On the other hand, Lemma 3.5 states that $\lambda_{i}(k)$ tends to $\lambda_{i}$ as $k \rightarrow 0$. Consequently there exists a one-to-one correspondence between each $\lambda_{i}$ such that $0<\lambda_{i} \leqq 1$ and $k_{i}$ such that $\lambda_{i}\left(k_{i}\right)=1$. Clearly $E_{i}=-k_{i}^{2}$ is the energy level of the bound state created by the potential $|V|$. If $\lambda_{i}$ equals unity, that is $E_{i}=0$, the zero-energy "bound state" is a true bound state if and only if $l+(n-3) / 2>\frac{1}{2}$ (cf. the boundary condition (3.7)).

Finally we arrive at the main theorem:

Theorem 3.2. For $2 l+n-2 \geqq 1$, the number of bound states, $N_{n}^{l}$, produced by the potential $V$, satisfying (2.5), in the l-th wave in the $n$ dimensional space satisfies the inequality

$$
N_{n}^{l}<\frac{1}{2 l+n-2} \int_{0}^{\infty} r|V(r)| d r
$$

Proof: By means of Theorem 3.1, $N_{n}^{l}$ is equal to or smaller than the number of eigenvalues of $G_{n}^{l}$ lying on the interval $(0,1]$. Let $\lambda_{N} \leqq 1<\lambda_{N+1}$, then

$$
N_{n}^{l} \leqq N \leqq \sum_{i=1}^{N} \frac{1}{\lambda_{i}}<\sum_{i=1}^{\infty} \frac{1}{\lambda_{i}}=\frac{1}{2 l+n-2} \int_{0}^{\infty} r|V(r)| d r
$$

The inequality (3.11) is the generalized Bargmann's inequality. It must be noticed that each bound state has the multiplicity $h(n, l)$, if we take into account the freedom of the angular part.

\section{$\S 4$. One-Dimensional $\mathbb{C a s e}$}

The bound state problem in the one-dimensional space is to solve the eigenvalue equation

$$
\left.\left[-\left(\frac{d}{d x}\right)^{2}+V(x)\right] \psi_{1}(x)=E \psi_{1}(x),-\infty\right)<x<\infty
$$

for which the eigenfunctions belong to $L^{2}((-\infty, \infty) ; d x)$. If we assume 
that the potential $V$ is a real-valued piecewise-continuous function satisfying the restriction

$$
\int_{-\infty}^{\infty}(|x|+1)|V(x)| d x<\infty
$$

the differential operator on the left hand side of Eq. (4.1) turns out to be essentially self-adjoint and point eigenvalues of this operator, if any, are all negative [5].

Suggested by the results in the previous section, we shall here make the following assumption:

Assumption 4. H. The number of bound states of Eq. (4.1), which is equal to the number of point eigenvalues because of no degeneracy, is not greater than the number of point eigenvalues $\lambda$ lying on the interval $[0,1)$ of the eigenvalue equation

$$
\left[-\left(\frac{d}{d x}\right)^{2}-\lambda|V(x)|\right] \psi_{1}(x)=0,-\infty<x<\infty
$$

for which the eigenfunctions belong to the Hilbert space $\mathscr{H}_{V}^{1} \equiv L((-\infty, \infty)$; $|V(x)| d x)$.

This assumption will be proved in Sec. 6 .

In the one-dimensional case, we shall mainly confine ourselves to $\mathscr{H}_{V}^{1}$, so we shall write $d \mu_{1}(x)$ instead of $|V(x)| d x$ and the inner product, the norm of vectors, the Hilbert-Schmidt norm, and the trace of operators in $\mathscr{H}_{V}^{1}$ by $(,)_{1,1}\|\|_{1,1}\left\|_{1}\right\|_{2}$, and $\operatorname{tr}_{1}($ ), respectively.

Since any eigenfunction $\psi_{1}$ of Eq. (4.3) satisfies the boundary condition

$$
\phi_{1}(x)=O(1), \quad x \rightarrow \pm \infty,
$$

we are immediately led to

Lemma 4.1. The eigenvalue equation (4.3) is equivalent to the integral equation

$$
\psi_{1}=c \cdot e_{0}+\lambda K_{1} \cdot \psi_{1} \quad(c: \text { constant })
$$

on $\mathscr{H}_{V}^{1}$ with a supplementary condition 


$$
\lambda\left(e_{0}, \psi_{1}\right)_{1}=0
$$

where the kernel function of the integral operator $K_{1}$ and the vector $e_{0}$ are given as

$$
\left(K_{1}\right)\left(x, x^{\prime}\right)=-\frac{1}{2}\left|x-x^{\prime}\right| \text { and }\left(e_{0}\right)(x) \equiv 1
$$

Proof: The proof is obtained, for example, by rewriting the differential equation (4.3) into its equivalent integral equations on the right-half and the left-half parts to the (say) origin of the real line separately and by making use of the continuity condition at the origin, that is, the solution clefined on the right- and left-half parts must be continuous up to the first derivatives at the origin.

From Eqs. (4.5) and (4.6), it readily follows that the vector $e_{0}$ is an eigenvector bolonging to the eigenvalue $\lambda=0$. For $\lambda \neq 0$, eigenvectors necessarily belong to $\mathscr{H}_{V}^{\frac{1}{V}}$, the orthogonal complement of $e_{0}$. Let $P$ be the projection operator from $\mathscr{H}_{V}^{1}$ to $\mathscr{H}_{V}^{\frac{1}{V}}$, then Eq. (4.5) takes the form

$$
\psi_{1}=\lambda P K_{1} P \psi_{1}
$$

on $\mathscr{H}_{V}^{\frac{1}{V}}$.

Lemma 4.2. The integral operator $P K_{1} P$ is of positive type and belongs to the trace class with its trace given by

$$
\operatorname{tr}_{1}\left(P K_{1} P\right)=\frac{\frac{1}{2} \int_{-\infty}^{\infty} \int_{-\infty}^{\infty}\left|x-x^{\prime}\right| d \mu_{1}\left(x^{\prime}\right) d \mu_{1}(x)}{\int_{-\infty}^{\infty} d \mu_{1}(x)}
$$

Proof: To prove the positivity, it is sufficient to show

$$
\int_{-\infty}^{\infty} f(x) \int_{-\infty}^{\infty}\left(-\frac{1}{2}\left|x-x^{\prime}\right|\right) f\left(x^{\prime}\right) d x^{\prime} d x
$$

is positive for not identically-vanishing, real-valued infinitely differentiable function $f$ with compact support in $(-\infty, \infty)$ satisfying the condition

$$
\int_{-\infty}^{\infty} f(x) d x=0
$$


For such a function, the expression (4.10) is transformed, by the integration by parts, into

$$
\int_{-\infty}^{\infty}\left(\int_{-\infty}^{x} f\left(x^{\prime}\right) d x^{\prime}\right)^{2} d x
$$

which is evidently positive.

In order to prove that $P K_{1} P$ has a trace (and hence is of HilbertSchmidt type because of Lemma 3.2), we note that the kernel function of $P K_{1} P$ is given by

$$
\begin{aligned}
&\left(P K_{1} P\right)\left(x, x^{\prime}\right)=\left(K_{1}\right)\left(x, x^{\prime}\right)-\frac{\left(K_{1} e_{0}\right)(x) \cdot e_{0}\left(x^{\prime}\right)}{\left(e_{0}, e_{0}\right)_{1}} \\
&-\frac{e_{0}(x) \cdot\left(K_{1} e_{0}\right)\left(x^{\prime}\right)}{\left(e_{0}, e_{0}\right)_{1}}+\frac{\left(e_{0}, K_{1} e_{0}\right)_{1}}{\left(e_{0}, e_{0}\right)_{1}^{2}} e_{0}(x) \circ e_{0}\left(x^{\prime}\right)
\end{aligned}
$$

From this expression we obtain

$$
\int_{-\infty}^{\infty}\left(P K_{1} P\right)(x, x) d \mu_{1}(x)=\frac{\left(e_{0},-K_{1} e_{0}\right)_{1}}{\left(e_{0}, e_{0}\right)_{1}}
$$

the right hand side of which is certainly finite. Consequently, by the same argument as in Lemma 3.2, the equality (4.9) follows.

By noticing that there exists one more point eigenvalue $\lambda=0$ in addition to those of $P K_{1} P$ and by using the same arguments as in the proof of Theorem 3.2, we arrive, under the Assumption 4.1, at the following result:

Theorem $\mathbb{4}_{\circ} \mathbb{1}$. The number of bound states, $N_{1}$, produced by the potential $V$, with the restriction (4.2), in the one-dimensional space satisfies the inequality

$$
N_{1}<1+\frac{\frac{1}{2} \int_{-\infty}^{\infty} \int_{-\infty}^{\infty}\left|x-x^{\prime}\right|\left|V\left(x^{\prime}\right)\right| d x^{\prime}|V(x)| d x}{\int_{-\infty}^{\infty}|V(x)| d x}
$$

Thus far we have not assumed the spherical symmetry of the potential. If we require this, that is,

$$
V(-x)=V(x) \equiv V(r), r=|x|
$$


eigenfunctions of Eq. (4.3) split into two classes; even functions of $x$ and odd functions of $x$. Denoting the projection operators from $\mathscr{H}_{V}^{1}$ to the subspace consisting of even functions and that of odd functions by $P_{1}^{0}$ and $P_{1}^{1}\left(=1-P_{1}^{0}\right)$, respectively, we find

\section{Lemma 4.3.}

$$
\operatorname{tr}_{1}\left(P_{1}^{0} P K_{1} P P_{1}^{0}\right)=\frac{\frac{1}{2} \int_{0}^{\infty} \int_{0}^{\infty}\left|r-r^{\prime}\right| d \mu_{1}\left(r^{\prime}\right) d \mu_{1}(r)}{\int_{0}^{\infty} d \mu_{1}(r)}
$$

and

$$
\operatorname{tr}_{1}\left(P_{1}^{1} P K_{1} P P_{1}^{1}\right)=\int_{0}^{\infty} r d \mu_{1}(r)
$$

Proof: From the meaning of $P, P_{1}^{0}$ and $P_{1}^{1}$, it follows

$$
P_{1}^{0} P=P P_{1}^{0} \text { and } P_{1}^{1} P=P P_{1}^{1}=P_{1}^{1}
$$

from which and the equalities

$$
\begin{aligned}
& \left(P_{1}^{0} K_{1} P_{1}^{0}\right)\left(x, x^{\prime}\right)=-(1 / 4)\left(\left|x-x^{\prime}\right|+\left|x+x^{\prime}\right|\right), \\
& \left(P_{1}^{1} K_{1} P_{1}^{1}\right)\left(x, x^{\prime}\right)=-(1 / 4)\left(\left|x-x^{\prime}\right|-\left|x+x^{\prime}\right|\right),
\end{aligned}
$$

readily follow Eqs. (4.13) and (4.14).

Since the eigenvector $e_{0}$ belongs to $P_{1}^{0} \mathscr{H}_{V}^{1}$, we have

Theorem 4.2. For the spherically symmetric potential in the onedimensioal space, the number $N_{1}^{0}$ of bound states with even eigenfunctions satisfies

$$
N_{1}^{0}<1+\frac{\frac{1}{2} \int_{0}^{\infty} \int_{0}^{\infty}\left|r-r^{\prime}\right|\left|V\left(r^{\prime}\right)\right| d r^{\prime}|V(r)| d r}{\int_{0}^{\infty}|V(r)| d r}
$$

while the number $N_{1}^{1}$ of bound states with odd eigenfunctions satisfies

$$
N_{\mathrm{j}}^{1}<\int_{0}^{\infty} r|V(r)| d r
$$


[N. B.] In the previous section we have obtained the solutions of Eq. (3.7) satisfying the boundary condition $\phi_{n}^{l}(r)=O\left(r^{l+(n-1) / 2}\right)$ at the origin. If we put formally $n=1$ in Eq. (3.7), we get a centrifugal potential $l(l-1) / r^{2}$. Since this term must be absent in the one-dimensional case, $l$ equals 0 or 1 . Because for $l=0(l=1)$, above boundary condition becomes $\phi_{1}^{0}(r)=O(1)\left(\phi_{1}^{1}(r)=O(r)\right), \phi_{1}^{0}\left(\phi_{1}^{1}\right)$ can be extended, continuously up to the first derivatives, as an even (odd) function to the interval $(-\infty, 0)$. This justifies the notations $N_{1}^{0}$ and $N_{1}^{1}$ as special cases of $N_{n}^{l}$ with $n=1, l=0$ and $n=1, l=1$. Incidentally, the multiplicity $h(n, l)$ defined in Eq. (2.1) has a corresponding property: $\lim _{n \rightarrow 1} h(n, 0)=1, \lim _{n \rightarrow 1} h(n, 1)=1$, and $\lim _{n \rightarrow 1} h(n, l)=0$ for $l \geqq 2$.

\section{§5. The Case $\ell=0$ in Twomimensional Space}

The only case left untouched is the case $l=0$ with $n=2$. As was done in the previous section, we shall again make

Assumption 5.1. The number of bound states generated by the potential $|V|$, satisfying the condition (2.6), in the zero-th wave in the twodimensional space is equal to the number of point eigenvalues $\lambda$, lying on the interval $[0,1)$, of the eigenvalue equation

$$
\left[-\left(\frac{d}{d r}\right)^{2}-\frac{1 / 4}{r^{2}}-\lambda|V(r)|\right] \phi_{2}^{0}(r)=0,
$$

on the Hilbert space $\mathscr{H}_{V}\left(\equiv L^{2}((0, \infty):|V(r)| d r)\right)$, that is, with the boundary condition

$$
\phi_{2}^{0}(r)=O\left(r^{1 / 2}\right), \quad \text { as } \quad r \rightarrow 0 \text { and } r \rightarrow \infty
$$

In terms of the original function $\phi_{2}^{0}(r)=r^{-1 / 2} \phi_{2}^{0}(r)$ (cf. Eq. (2.3)), Eq. (5.1) is expressed as

$$
\left[-\frac{1}{r} \frac{d}{d r}\left(r \frac{d}{d r}\right)-\lambda|V(r)|\right] \psi_{2}^{0}(r)=0
$$

with the boundary condition

$$
\psi_{2}^{0}(r)=O(1), \quad \text { as } \quad r \rightarrow 0 \quad \text { and } \quad r \rightarrow \infty \text {. }
$$


By the change of variable

$$
r=e^{t}, \quad(-\infty<t<\infty)
$$

Eq. (5.2) becomes

$$
\left[-\left(\frac{d}{d t}\right)^{2}-\lambda|W(t)|\right] u(t)=0,-\infty<t<\infty
$$

where we have put

$$
\psi_{2}^{0}(r)=u(t), \quad \text { and } \quad r^{2} I(r)=\Pi^{r}(t)
$$

The condition (2.6) on $I$ is expressed in terms of $I I$ as

$$
\int_{-\infty}^{\infty}(|t|+1)\left|W^{\prime}(t)\right| d t<\infty
$$

while the boundary condition becomes

$$
u(t)=O(1), \quad \text { as } \quad t \rightarrow \pm \infty .
$$

By making use of the correspondences between (5.3) and (4.3), (5.4) and (4.2), and (5.5) and (4.4), we immediately find

Theorem 5.1. The number of bound states, $N_{2}^{0}$, produced by the potential $V$, under the restriction (2.6), in the zero-th wave in the twodimensional space, satisfies

$$
N_{2}^{0}<1+\frac{\frac{1}{2} \int_{0}^{\infty} r \int_{0}^{\infty} r^{\prime}\left|\log \left(r / r^{\prime}\right)\right|\left|V\left(r^{\prime}\right)\right| d r^{\prime}|V(r)| d r}{\int_{0}^{\infty} r|V(r)| d r} .
$$

Proof: From the inequality (4.12), we obtain

$$
N_{2}^{0}<1+\frac{\frac{1}{2} \int_{-\infty}^{\infty} \int_{-\infty}^{\infty}\left|t-t^{\prime}\right|\left|W\left(t^{\prime}\right)\right| d t^{\prime}|W(t)| d t}{\int_{-\infty}^{\infty}|W(t)| d t}
$$

This inequality, expressed in term of the variable $r$, takes the form of (5.6). 
Newton [3] has obtained the same inequality as (5.6) for the case $l=-\frac{1}{2}$ with $n=3$. Since in both cases $2 l+n-2$ vanishes, the relation between them is obvious.

Finally we note that, as is easily verified from Eq. (4.11), the kernel function of the integral operator corresponding to Eq. (5.1) is given, on the orthogonal complement of the eigenfunction with the eigenvalue $\lambda=0$, by

$$
\left(r r^{\prime}\right)^{1 / 2}\left\{-\frac{1}{2}\left|\log \left(r / r^{\prime}\right)\right|+\frac{\frac{1}{2} \int_{0}^{\infty} s|\log (r / s)| d \mu(s)}{\int_{0}^{\infty} s d \mu(s)}\right.
$$

$$
\left.+\frac{\frac{1}{2} \int_{0}^{\infty} s\left|\log \left(r^{\prime} / s\right)\right| d \mu(s)}{\int_{0}^{\infty} s d \mu(s)}-\frac{\frac{1}{2} \int_{0}^{\infty} s \int_{0}^{\infty} t|\log (s / t)| d \mu(t) d \mu(s)}{\left(\int_{0}^{\infty} s d \mu(s)\right)^{2}}\right\} .
$$

\section{§6. Proof of Assumptions 4. I and 5.1}

First we shall be concerned with the one-dimensional case. The following lemma is obvious:

Lemma 6.1. For $k>0$, the bound state problem

$$
\left[-\left(\frac{d}{d x}\right)^{2}-\lambda|V(x)|\right] \phi_{1}(x)=-k^{2} \psi_{1}(x), \quad-\infty<x<\infty,
$$

with the condition (4.2) on the potential function, is equivalent to the integral equation

$$
\psi_{1}=\lambda K_{1}(k) \psi_{1}
$$

on the Hilbert space $\mathscr{H}_{V}^{1}$, where the kernel function of the integral operator is given by

$$
K_{1}(k)\left(x, x^{\prime}\right)=\frac{1}{2 k} e^{-k\left|x-x^{\prime}\right|}
$$

The operator $K_{1}(k)$ is of positive type and has a trace given by 


$$
\operatorname{tr}_{1}\left(K_{1}(k)\right)=\frac{1}{2 k} \int_{-\infty}^{\infty}|V(x)| d x
$$

The difficulty in the one-dimensional case lies in the bad behaviour of $K_{1}(k)$ in the limit $k \rightarrow 0$. To avoid this singularity we shall consider, instead of Eq. (6.1), the following integral equation:

$$
f_{1}=\tau k_{1}(k) f_{1},
$$

with the kernel function

$$
k_{1}(k)\left(x, x^{\prime}\right)=e^{-k\left|x-x^{\prime}\right|}\left(\equiv 2 k K_{1}(k)\left(x, x^{\prime}\right)\right),
$$

and its formal $k \rightarrow 0$ limit:

$$
f_{1}=\tau k_{1} f_{1}
$$

with the kernel function

$$
k_{1}\left(x, x^{\prime}\right) \equiv 1
$$

We shall now prove

Lemma 6.2. The minimum eigenvalue $\tau_{0}(k)$ of Eq. (6.4) satisfies

$$
\frac{1}{\tau_{0}}-k \frac{\int_{-\infty}^{\infty} \int_{-\infty}^{\infty}\left|x-x^{\prime}\right| d \mu_{1}\left(x^{\prime}\right) d \mu_{1}(x)}{\int_{-\infty}^{\infty} d \mu_{1}(x)} \leqq \frac{1}{\tau_{0}(k)}<\frac{1}{\tau_{0}},
$$

where $\tau_{0}$ is the (unique) eigenvalue of $E q .(6.4)_{0}$ :

$$
\frac{1}{\tau_{0}}=\int_{-\infty}^{\infty} d \mu_{1}(x)
$$

Proof: The equality (6.7) is obvious. Since $\tau_{0}(k)$ satisfies

$$
\frac{1}{\tau_{0}(k)}<\operatorname{tr}_{1}\left(k_{1}(k)\right)=\int_{-\infty}^{\infty} d \mu_{1}(x)=\frac{1}{\tau_{0}}
$$

we have only to show the left half of the inequality (6.6). Through the maximum principle, it follows 


$$
\begin{aligned}
\frac{1}{\tau_{0}(k)} & =\max _{f \in \mathscr{H} \frac{1}{V}} \frac{\left(f, k_{1}(k) f\right)_{1}}{(f, f)_{1}} \geqq \frac{\int_{-\infty}^{\infty} \int_{-\infty}^{\infty} k_{1}(k)\left(x, x^{\prime}\right) d \mu_{1}\left(x^{\prime}\right) d \mu_{1}(x)}{\int_{-\infty}^{\infty} d \mu_{1}(x)} \\
& =\int_{-\infty}^{\infty} d \mu_{1}(x)-\frac{\int_{-\infty}^{\infty} \int_{-\infty}^{\infty}\left(1-e^{-k\left|x-x^{\prime}\right|}\right) d \mu_{1}\left(x^{\prime}\right) d \mu_{1}(x)}{\int_{-\infty}^{\infty} d \mu_{1}(x)} \\
& \geqq \frac{1}{\tau_{0}}-k \frac{\int_{-\infty}^{\infty} \int_{-\infty}^{\infty}\left|x-x^{\prime}\right| d \mu_{1}\left(x^{\prime}\right) d \mu_{1}(x)}{\int_{-\infty}^{\infty} d \mu_{1}(x)} .
\end{aligned}
$$

In the first inequality we have cliosen the eigenfunction of Eq. $(6.4)_{0}$ $\left(f_{1}(x) \equiv 1\right)$ as a trial function, and at the final inequality we have used the obvious relation $1-e^{-k\left|x-x^{\prime}\right|} \leqq k\left|x-x^{\prime}\right|$.

Lemma 6.3. Let $P(k)$ be the projection operator from $\mathscr{H}_{V}^{1}$ to the orthogonal complement of the eigenvector of Eq. (6.4) with the eigenvalue $\tau_{0}(k)$. Then $P(k) K_{1}(k) P(k)$ is a positive operator and has a trace uniformly bounded with respect to $k(>0)$.

Proof: The positivity of $P(k) K_{1}(k) P(k)$ is obvious. From the equality

$$
k_{1}(k)=\frac{1}{\tau_{0}(k)}(1-P(k))+P(k) k_{1}(k) P(k),
$$

and (6.6), it follows

$$
\operatorname{tr}_{1}\left(P(k) k_{1}(k) P(k)\right)=\operatorname{tr}_{1}\left(k_{1}(k)\right)-\frac{1}{\tau_{0}(k)}=\frac{1}{\tau_{0}}-\frac{1}{\tau_{0}(k)}
$$

$$
\leqq k \frac{\int_{-\infty}^{\infty} \int_{-\infty}^{\infty}\left|x-x^{\prime}\right| d \mu_{1}\left(x^{\prime}\right) d \mu_{1}(x)}{\int_{-\infty}^{\infty} d \mu_{1}(x)}
$$

and hence

$$
\operatorname{tr}_{1}\left(P(k) K_{1}(k) P(k)\right)=\frac{1}{2 k} \operatorname{tr}_{1}\left(P(k) k_{1}(k) P(k)\right)
$$

$$
\leqq \frac{\frac{1}{2} \int_{-\infty}^{\infty} \int_{-\infty}^{\infty}\left|x-x^{\prime}\right| d \mu_{1}\left(x^{\prime}\right) d \mu_{1}(x)}{\int_{-\infty}^{\infty} d \mu_{1}(x)}
$$


Lemma 6.4. Let $P$ be the projection operator from $\mathscr{H}_{V}^{1}$ to the orthogonal complement of the eigenvector of Eq. (6.4) 0 with the eigenvalue $\tau_{0}$, Then

$$
{ }_{1}\|P(k)-P\|_{2}=o\left(k^{1 / 2}\right), \quad \text { as } k \rightarrow 0 \text {. }
$$

Proof: From liq. (6.8) and the equality

$$
h_{1}=\frac{1}{\tau_{0}}\left(1-P^{p}\right)
$$

we obtain

$$
\begin{aligned}
P(k)-P & =\tau_{0} k_{1}-\tau_{0}(k) k_{1}(k)+\tau_{0}(k) P(k) k_{1}(k) P(k) \\
& =\tau_{0}\left(k_{1}-k_{1}(k)\right)+\left(\tau_{0}-\tau_{0}(k)\right) k_{1}(k)+\tau_{0}(k) P(k) k_{1}(k) P(k)
\end{aligned}
$$

Consequently

$$
\begin{aligned}
{ }_{1}\|P(k)-P\|_{2} \leqq \tau_{0} \cdot{ }_{1}\left\|k_{1}-k_{1}(k)\right\|_{2}+\left|\tau_{0}-\tau_{0}(k)\right| \cdot{ }_{1}\left\|k_{1}(k)\right\|_{2} \\
+\tau_{0}(k) \cdot{ }_{1}\left\|P(k) k_{1}(k) P(k)\right\|_{2} .
\end{aligned}
$$

As is easily seen from (6.6) and (6.9), the second and third terms in the right hand side of this inequality are of order $O(k)$ as $k \rightarrow 0$. As for the first term

$$
\begin{array}{r}
{ }_{1}\left\|k_{1}-k_{1}(k)\right\|_{2}^{2}=\int_{-\infty}^{\infty} \int_{-\infty}^{\infty}\left(1-e^{-k\left|x-x^{\prime}\right|}\right)^{2} d \mu_{1}\left(x^{\prime}\right) d \mu_{1}(x) \\
\leqq k \int_{-\infty}^{\infty} \int_{-\infty}^{\infty}\left|x-x^{\prime}\right|\left(1-e^{-k\left|x-x^{\prime}\right|}\right) d \mu_{1}\left(x^{\prime}\right) d \mu_{1}(x),
\end{array}
$$

the last of which is evidently of order $o(k)$.

Now we can prove

Theorem 6.1. The operator $P(k) K_{1}(k) P(k)$ approaches as $k \rightarrow 0$ to the operator $P K_{1} P$ defined in Sec. 4 in the Hilbert-Schmidt norm.

Proof: We first note that the projection operator $P$ defined in Lemma 6.4 is identical with the projection operator introduced in Eq. (4.8). Because of the equality 


$$
\begin{aligned}
& P(k) K_{1}(k) P(k)-P K_{1} P=P\left(K_{1}(k)-K_{1}\right) P+(P(k)-P) K_{1}(k) P(k) \\
& \quad+P(k) K_{1}(k)(P(k)-P)-(P(k)-P) K_{1}(k)(P(k)-P),
\end{aligned}
$$

we obtain

$$
\begin{aligned}
& { }_{1}\left\|P(k) K_{1}(k) P(k)-P K_{1} P\right\|_{2} \leqq{ }_{1}\left\|P\left(K_{1}(k)-K_{1}\right) P\right\|_{2} \\
& +2{ }_{1}\|P(k)-P\|_{2}{ }^{\circ}{ }_{1}\left\|P(k) K_{1} P(k)\right\|_{1}+{ }_{1}\|P(k)-P\|_{2}{ }^{\circ}{ }_{1}\left\|K_{1}(k)\right\|_{2},
\end{aligned}
$$

where use has been made of the equality $P(k) K_{1}(k)=K_{1}(k) P(k)=$ $P(k) K_{1}(k) P(k)$. From Lemma 6.3 and 6.4 it immediately follows that the second and third terms on the right hand side tend to zero as $k \rightarrow 0$. To prove ${ }_{1}\left\|P\left(K_{1}(k)-K_{1}\right) P\right\|_{2} \rightarrow 0$, we shall first derive an inequality for the kernel function of $P K_{1}(k) P$. The positivity of $P K_{1}(k) P$ implies (cf. (3.4))

$$
\left(P K_{1}(k) P\right)\left(x, x^{\prime}\right)^{2} \leqq\left(P K_{1}(k) P(x, x) \cdot\left(P K_{1}(k) P\right)\left(x^{\prime}, x^{\prime}\right),\right.
$$

where $\left(P K_{1}(k) P\right)(x, x)$ is shown to be equal to

$$
\tau_{0} \cdot \int_{-\infty}^{\infty} \frac{1-e^{-k|x-y|}}{k} d \mu_{1}(y)-\tau_{0}^{2} \cdot \int_{-\infty}^{\infty} \int_{-\infty}^{\infty} \frac{1-e^{-k|y-z|}}{2 k} d \mu_{1}(y) d \mu_{1}(z) .
$$

As is easily verified this is dominated by

$$
\tau_{0} \cdot \int_{-\infty}^{\infty}|x-y| d \mu_{1}(y)
$$

which is certainly integrable. In addition, the kernel function of $P\left(K_{1}(k)-K_{1}\right) P$ is

$$
\begin{gathered}
\left(P\left(K_{1}(k)-K_{1}\right) P\right)\left(x, x^{\prime}\right)=h\left(x, x^{\prime} ; k\right)-\frac{\int_{-\infty}^{\infty} h(x, y ; k) d \mu_{1}(y)}{\int_{-\infty}^{\infty} d \mu_{1}(y)} \\
-\frac{\int_{-\infty}^{\infty} h\left(y, x^{\prime} ; k\right) d \mu_{1}(y)}{\int_{-\infty}^{\infty} d \mu_{1}(y)}+\frac{\int_{-\infty}^{\infty} \int_{-\infty}^{\infty} h(y, z ; k) d \mu_{1}(y) d \mu_{1}(z)}{\left(\int_{-\infty}^{\infty} d \mu_{1}(y)\right)^{2}}
\end{gathered}
$$

where 


$$
h\left(x, x^{\prime} ; k\right)=\frac{1}{2 k}\left(e^{-k\left|x-x^{\prime}\right|}-1+k\left|x-x^{\prime}\right|\right),
$$

approaches zero pointwise as $k \rightarrow 0$. Therefore it follows that

$$
{ }_{1}\left\|P\left(K_{1}(k)-K_{1}\right) P\right\|_{2} \rightarrow 0,
$$

which implies

$$
{ }_{1}\left\|P(k) K_{1}(k) P(k)-P K_{1} P\right\|_{2} \rightarrow 0, \quad \text { as } \quad k \rightarrow 0 .
$$

By Theorem 6.1 and Lemma 3.5, it is shown that each eigenvalue $\lambda_{i}(k)(i=1,2, \ldots)\left(0<\lambda_{1}(k)<\lambda_{2}(k)<\cdots\right)$ of $P(k) K_{1}(k) P(k)$ approaches the eigenvalue $\lambda_{i}\left(0<\lambda_{1}<\lambda_{2}<\cdots\right)$ of $P K_{1} P$ as $k \rightarrow 0$. Moreover the smallest eigenvalue $\lambda_{0}(k) \equiv 2 k \tau_{0}(k)$ of $K_{1}(k)$ tends to zero, which is equal to the smallest eigenvalue of Eqs. (4.5) and (4.6). Since it can also be shown that each $\lambda_{i}(k)(i=0,1,2, \ldots)$ is a continuous, monotonously increasing function of $k$ and tends to infinity as $k \rightarrow \infty$, we can justify Assumption 4.1 by the same method as in the proof of Theorem 3.1.

The Assumption 5.1 can be proved analogously, although calculations are slightly complicated. We shall briefly sketch the way of justification.

Lemma 6.1'. For $k>0$, the bound state problem (2.8) for $l=0$, $n=2$, with the condition (2.6) on the potential, is equivalent to the integral equation

$$
\phi_{2}^{0}=\lambda G_{2}^{0}(k) \phi_{2}^{0}
$$

on the Hilbert space $\mathscr{H}_{V}$, where the kernel function of the integral operator $G_{2}^{0}(k)$ is given by

$$
G_{2}^{0}(k)\left(r, r^{\prime}\right)=\left(r r^{\prime}\right)^{1 / 2} \times\left\{\begin{array}{l}
I_{0}(k r) K_{0}\left(k r^{\prime}\right), 0 \leqq r<r^{\prime} \\
I_{0}\left(k r^{\prime}\right) K_{0}(k r), r>r^{\prime}
\end{array}\right.
$$

The operator $G_{2}^{0}(k)$ is positive and belongs to the trace class with its trace given by

$$
\operatorname{tr}\left(G_{2}^{0}(k)\right)=\int_{0}^{\infty} r I_{0}(k r) K_{0}(k r)|V(r)| d r
$$


Proof: All but the positivity of $G_{2}^{0}(k)$ are obvious. Although the differential operator $-(d / d r)^{2}-1 / 4 r^{2}+k^{2}$ contains the negative term $-1 / 4 r^{2}$, this operator can be transformed into $-(d / d t)^{2}+k^{2} e^{2 t}$ by a unitary transformation, as was done in the previous section. Therefore by the same argument as in Lemma 3.2, the positivity of $G_{2}^{0}(k)$ follows.

As will be shown in Appendix $A$, the trace of $C_{2}^{0}(h)$ behaves in the limit $k \rightarrow 0$ as

$$
\lim _{k \rightarrow+0}\left(\operatorname{tr}\left(G_{2}^{0}(k)\right)+\log k \int_{0}^{\infty} d \mu(r)\right)=-\int_{0}^{\infty}(\gamma+\log (r / 2)) r d \mu(r)
$$

( $\gamma$; Euler's constant)

Since $\operatorname{tr}\left(G_{2}^{0}(k)\right)$ diverges when $k$ tends to zero, we must extract from $G_{2}^{0}(k)$ the diverging part. For this purpose we shall consider the integral equation

$$
f_{2}^{0}=\tau g_{2}^{0}(k) f_{2}^{0}
$$

with the kernel function

$$
g_{2}^{0}(k)\left(r, r^{\prime}\right)=\frac{1}{-\log k} G_{2}^{0}(k)\left(r, r^{\prime}\right)
$$

and its formal $k \rightarrow 0$ limit:

$$
f_{2}^{0}=\tau g_{2}^{0} f_{2}^{0}
$$

with the kernel function

$$
g_{2}^{0}\left(r, r^{\prime}\right)=\left(r r^{\prime}\right)^{1 / 2}
$$

As a lemma corresponding to Lemma 6.2, we obtain

Lemma 6.2'. The minimum eigenvalue $\tilde{\tau}_{0}(k)$ of Eq. $(6.4)^{\prime}$ satisfies, for sufficiently small $k$,

$(6.6)^{\prime}\left(\begin{array}{l}\frac{1}{\tilde{\tau}_{0}}+\frac{1}{\log k} \int_{0}^{\infty}(\gamma+\log (r / 2)) r d \mu(r)+o\left(\frac{1}{|\log k|}\right) \\ \geqq \operatorname{tr}\left(g_{2}^{0}(k)\right)>\frac{1}{\tilde{\tau}_{0}(k)}\end{array}\right.$ 


$$
\mid \geqq \frac{1}{\tilde{\tau}_{0}}+\frac{1}{\log k} \frac{2 \int_{0}^{\infty}(\gamma+\log (r / 2)) r \int_{0}^{r} r^{\prime} d \mu\left(r^{\prime}\right) d \mu(r)}{\int_{0}^{\infty} r d \mu(r)}+o\left(\frac{1}{|\log k|}\right),
$$

where $\tilde{\tau}_{u}$ is the (unique) eigenvalue of Eq. $(6.4)_{0}^{\prime}$ :

$$
\frac{1}{\tilde{\tau}_{0}}=\int_{0}^{\infty} r d \mu(r)
$$

Proof: The first inequality in $(6.6)^{\prime}$ is obvious (cf. Eq. $(6.3)^{\prime}$ ), so is the second inequality. The last inequality will be proved in Appendix B.

Lemma 6.3'. Let $\tilde{P}(k)$ be the projection operator from $\mathscr{H}_{V}$ to the orthogonal complement of the eigenvector of Eq. (6.4)' with the eigenvalue $\tilde{\tau}_{0}(k)$. Then $\tilde{P}(k) G_{2}^{0}(k) \tilde{P}(k)$ is of positive type and has a trace uniformly bounded even in the limit $k \rightarrow 0$.

Proof: By the same reasoning as in Lemma 6.3, we obtain from $(6.6)^{\prime}$

$$
\begin{aligned}
\operatorname{tr} & \left(\tilde{P}(k) G_{2}^{0}(k) \tilde{P}(k)\right)=-\log k \cdot \operatorname{tr}\left(\tilde{P}(k) g_{2}^{0}(k) \tilde{P}(k)\right) \\
& =-\log k\left(\operatorname{tr}\left(g_{2}^{0}(k)\right)-\frac{1}{\tilde{\tau}_{0}(k)}\right) \\
& \leqq \frac{\frac{1}{2} \int_{0}^{\infty} r \int_{0}^{\infty} r^{\prime}\left|\log \left(r / r^{\prime}\right)\right| d \mu\left(r^{\prime}\right) d \mu(r)}{\int_{0}^{\infty} r d \mu(r)}+\varepsilon(k),(\varepsilon(k) \rightarrow 0, k \rightarrow 0) .
\end{aligned}
$$

As will be proved in Appendix $\mathrm{C}$, the following also holds:

Lemma 6.4'. Let $\widetilde{P}$ be the projection operator from $\mathscr{H}_{V}$ to the orthogonal complement of the eigenvector of Eq. (6.4) $)_{0}^{\prime}$ with the eigenvalue $\tilde{\tau}_{0}$. Then

$$
\|\tilde{P}(k)-\tilde{P}\|_{2}=o\left(\frac{1}{|\log k|^{1 / 2}}\right), \quad \text { as } \quad k \rightarrow 0
$$

Finally we have

Theørem 6.1'. The integral operator $\tilde{P}(k) G_{2}^{0}(k) \tilde{P}(k)$ approaches as 
$k \rightarrow 0$ to the integral operator whose kernel function is given by (5.7) in the Hilbert-Schmidt norm.

Proof: Through the same argument as in the proof of Theorem 6.1, it is sufficient to show that $\dot{P} G_{2}^{0}(k) P$ approaches the above limit in the Hilbert-Schmidt norm. From the estimate of the kernel function of $\tilde{P} G_{2}^{0}(k) \tilde{P}$ given in Appendix D, it follows that we can make use of Lebesgue's dominated convergence theorem in the evaluation of the Hilbert-Schmidt norm of the difference between two operators. Moreover, by using the estimates obtained in Appendix B, we can confirm that the kernel function $\left(P G_{2}^{0}(k) P\right)\left(r, r^{\prime}\right)$ tends, for each (non-zero finite) $r$ and $r^{\prime}$, to

$$
\begin{aligned}
& \left(r r^{\prime}\right)^{1 / 2}\left\{-\log \left[\max \left(r, r^{\prime}\right)\right]+\frac{\int_{0}^{\infty} s \cdot \log [\max (r, s)] d \mu(s)}{\int_{0}^{\infty} s d \mu(s)}\right. \\
& \left.+\frac{\int_{0}^{\infty} s \cdot \log \left[\max \left(r^{\prime}, s\right)\right] d \mu(s)}{\int_{0}^{\infty} s d \mu(s)}-\frac{\int_{0}^{\infty} s \int_{0}^{\infty} t \cdot \log [\max (s, t)] d \mu(t) d \mu(s)}{\left(\int_{0}^{\infty} s d \mu(s)\right)^{2}}\right\},
\end{aligned}
$$

which is shown, by a simple manipulation, to be equal to the expression (5.7).

Thus Assumption 5.1 can be justified by the same reasoning as in the justification of Assumption 4.1.

\section{§7. Summary and Discussion}

We have generalized Bargmann's inequality to the space of arbitrary dimension in a mathematically rigorous way. The number of bound states, created in the $l$-th partial wave in the $n$-dimensional space, by a spherically symmetric potential satisfies the inequality (3.11) for the case $2 l+n-2 \geqq 1$ under the restriction (2.5) on the potential function. The inequality can be written in a compact form:

$$
N_{n}^{l}<\operatorname{tr}_{n}\left(P_{n}^{l} K_{n}\right) \quad(2 l+n-2 \geqq 1) .
$$

Here $P_{n}^{l}$ is the projection operator defined on $\mathscr{H}_{V}^{n}=L^{2}\left(R^{n} ; V(|\vec{x}|) d \vec{x}\right)$ 
such that $P_{n}^{l} \Lambda_{n}^{2} P_{n}^{l}=-l(l+n-2) P_{n}^{l}$, and $K_{n}$ is the integral operator on $\mathscr{H}_{V}^{n}$, whose kernel function $\left(K_{n}\right)\left(\vec{x}, \vec{x}^{\prime}\right)$ is given by $\left(\Gamma(n / 2) / 2(n-2) \pi^{n / 2}\right)$ $\left|\vec{x}-\vec{x}^{\prime}\right|^{2-n}(n \geqq 3)$ or $(1 / 2 \pi) \cdot \log \left(1 /\left|x-\vec{x}^{\prime}\right|\right)(n=2)$, that is an elementary solution of $-\Delta_{n}$, and $\operatorname{tr}_{n}()$ means the trace on $\mathscr{H}_{V}^{n}$. All remain true for the case $l=1, n=1$; in Sec. 4 we have already introduced $P_{1}^{1}$ and $K_{1}$, and the corresponding inequality (4.16) is rewritten as $N_{1}^{1}<\operatorname{tr}_{1}\left(P_{1}^{1} K_{1}\right)$. For $2 l+n-2 \leqq 0$, i.e., $l=0, n=2$ and $l=0, n=1$, under the condition (2.6) and (4.2), respectively, the inequalities (5.6) and (4.15) can be expressed as

$$
N_{n}^{l}<1+\operatorname{tr}_{n}\left(P P_{n}^{l} K_{n}\right), \quad(2 l+n-2 \leqq 0)
$$

where $P$ is the projection operator from $\mathscr{H}_{V}^{n}$ to the orthogonal complement of the vector $e_{0}\left(e_{0}(\vec{x}) \equiv 1\right.$ in both cases $)$.

As was noticed in Sec. 2, inequalities in which $|V|$ is replaced by $\left|V_{-}\right|$hold true also.

The saturation of the inequalities, established for $n=3$ in Refs. [1] and $[2]$, can be proved analogously, that is, we can find a potential such that the generalized Bargmann's inequality takes the form $N_{n}^{l}<m+\varepsilon$ ( $m$ is an arbitrary positive integer and $\varepsilon$ is an arbitrary positive number) and the potential produces just $m$ bound states in the $l$-th wave in the $n$-dimensional space. Moreover we can prove that, for the case $2 l+n-2 \leqq 0$, if the potential function is non-positive (and, of course, not identically vanishing) there emerges at least one bound state: $N_{n}^{l} \geqq 1$.

\section{Acknowledgement}

The author expresses his hearty thanks to Prof. H. Araki for his kind interest and his critical comments on the lack of mathematical rigour in the original manuscript. He also thanks to Dr. T. Yoshida for his careful reading of the manuscript.

\section{Appemdix $\mathbb{A}:$ Proof of $(6.3)^{\prime}$}

From the well-known behaviours of $I_{0}$ and $K_{0}$ near the origin and the point at infinity: 


$$
\left.\begin{array}{l}
I_{0}(x)=1+O\left(x^{2}\right), \\
K_{0}(x)=-(\gamma+\log (x / 2))+O\left(x^{2} \log x\right),
\end{array}\right\} x \sim 0
$$

and

$$
\left.\begin{array}{l}
I_{0}(x)=\frac{e^{x}}{(2 \pi x)^{1 / 2}}\left(1+O\left(x^{-2}\right)\right), \\
K_{0}(x)=\left(\frac{\pi}{2 x}\right)^{1 / 2} e^{-x}\left(1+O\left(x^{-2}\right)\right),
\end{array}\right\} x \sim \infty
$$

we can obtain the estimates

$$
I_{0}(k r)=e^{k r}+R_{1}(k r), \quad k r \geqq 0
$$

with

$$
\left|R_{1}(k r)\right| \leqq a_{1} \frac{k r}{1+k r} \cdot e^{k r} \quad\left(a_{1}: \text { constant }\right)
$$

and

with

$$
\left.\begin{array}{r}
K_{0}(k r)=-(\log k+\gamma+\log (r / 2)) e^{-k r}+R_{2}(k r), \\
k r>0 \\
\left|R_{2}(k r)\right| \leqq a_{2} \frac{k r}{1+k r}(|\log k r|+1) e^{-k r} \\
\left(a_{2}: \text { constant }\right) .
\end{array}\right\}
$$

Therefore $\operatorname{tr}\left(G_{2}^{0}(k)\right)$ can be written as

$$
\begin{aligned}
& \operatorname{tr}\left(G_{2}^{0}(k)\right)=-\log k \int_{0}^{\infty} r d \mu(r)-\int_{0}^{\infty}(\gamma+\log (r / 2)) r d \mu(r) \\
& -\int_{0}^{\infty}(\gamma+\log (k r / 2)) e^{-k r} R_{1}(k r) r d \mu(r)+\int_{0}^{\infty} I_{0}(k r) R_{2}(k r) r d \mu(r) .
\end{aligned}
$$

To prove that the last two terms tend to zero as $k \rightarrow 0$, it is sufficient to show

$$
\lim _{k \rightarrow+0} k|\log k| \int_{0}^{\infty} \frac{r^{2}}{1+k r} d \mu(r)=0
$$

By dividing the integral into two parts: 


$$
\int_{0}^{k^{-1 / 2}} \frac{r^{2}}{1+k r} d \mu(r)+\int_{k^{-1 / 2}}^{\infty} \frac{r^{2}}{1+k r} d \mu(r)
$$

we find

$$
\begin{aligned}
\int_{0}^{k^{-1 / 2}} \frac{r^{2}}{1+k r} d \mu(r) & =\int_{0}^{k^{-1 / 2}} \frac{k r^{1 / 2}}{1+k r} \frac{r}{k^{1 / 2}} d \mu(r) \leqq \frac{1}{k^{1 / 2}} \int_{0}^{k^{-1 / 2}} r d \mu(r) \\
& \leqq \frac{1}{k^{1 / 2}} \int_{0}^{\infty} r d \mu(r),
\end{aligned}
$$

and

$$
\begin{aligned}
& \int_{k^{-1 / 2}}^{\infty} \frac{r^{2}}{1+k r} d \mu(r)=\frac{1}{k} \int_{k^{-1 / 2}}^{\infty} \frac{k r}{1+k r} r d \mu(r) \leqq \frac{1}{k} \int_{k^{-1 / 2}}^{\infty} r d \mu(r) \\
& \quad \leqq \frac{1}{k} \int_{k^{-1 / 2}}^{\infty} r \frac{|\log r|}{\left|\log \left(k^{-1 / 2}\right)\right|} d \mu(r)=\frac{2}{k|\log k|} \int_{k^{-1 / 2}}^{\infty} r|\log r| d \mu(r)
\end{aligned}
$$

From these estimates (A.3) immediately follows.

\section{Appendix B : Proof of Lemma 6.2}

The maximum principle states that

$$
\begin{aligned}
\frac{1}{\tilde{\tau}_{0}(k)} & =\max _{f \in \mathscr{C}_{V}} \frac{\left(f, g_{2}^{0}(k) f\right)}{(f, f)} \\
& \geqq \frac{2}{-\log k} \frac{\int_{0}^{\infty} r K_{0}(k r) \int_{0}^{r} r^{\prime} I_{0}\left(k r^{\prime}\right) d \mu\left(r^{\prime}\right) d \mu(r)}{\int_{0}^{\infty} r d \mu(r)}
\end{aligned}
$$

where we have chosen the eigenfunction of Eq. $(6.4)_{0}^{\prime}\left(f_{2}^{0}(r)=r^{1 / 2}\right)$ as a trial function. By the use of (A.1) and (A.2), the numerator of the last expression is written as

$$
\begin{aligned}
& \int_{0}^{\infty} r K_{0}(k r) \int_{0}^{r} r^{\prime} I_{0}\left(k r^{\prime}\right) d \mu\left(r^{\prime}\right) d \mu(r)=-\log k \int_{0}^{\infty} r e^{-k r} \int_{0}^{r} r^{\prime} e^{k r^{\prime}} d \mu\left(r^{\prime}\right) d \mu(r) \\
& -\int_{0}^{\infty} r(r+\operatorname{lng}(r / 2)) e^{-k r} \int_{0}^{r} r^{\prime} I_{0}\left(k r^{\prime}\right) d \mu\left(r^{\prime}\right) d \mu(r)
\end{aligned}
$$




$$
-\log k \int_{0}^{\infty} r e^{-k r} \int_{0}^{r} r^{\prime} R_{1}\left(k r^{\prime}\right) d \mu\left(r^{\prime}\right) d \mu(r)+\int_{0}^{\infty} r R_{2}(k r) \int_{0}^{r} r^{\prime} I_{0}\left(k r^{\prime}\right) d \mu\left(r^{\prime}\right) d \mu(r)
$$

The first term on the right hand side is estimated as

$$
\begin{aligned}
& \left|-\log k \int_{0}^{\infty} r e^{-k r} \int_{0}^{r} r^{\prime} e^{k r^{\prime}} d \mu\left(r^{\prime}\right) d \mu(r)+\log k \int_{0}^{\infty} r e^{-k r} \int_{0}^{r} r^{\prime} d \mu\left(r^{\prime}\right) d \mu(r)\right| \\
& \leqq(-\log k) c_{1} \int_{0}^{\infty} r e^{-k r} \int_{0}^{r} \frac{k r^{\prime 2}}{1+k r^{\prime}} e^{k r^{\prime}} d \mu\left(r^{\prime}\right) d \mu(r) \\
& \quad \text { (because } e^{\left.k r^{\prime}-1 \leqq c_{1} \frac{k r^{\prime}}{1+k r^{\prime}} e^{k r^{\prime}}\right)} \\
& \leqq c_{1}|\log k| \int_{0}^{\infty} r d \mu(r) \int_{0}^{\infty} \frac{k r^{\prime 2}}{1+k r^{\prime}} d \mu\left(r^{\prime}\right),
\end{aligned}
$$

which approaches zero as $k \rightarrow 0$ because of (A.3). (Note that the third term is incidentally shown to vanish as $k \rightarrow 0$.) In the same way, we obtain

$$
\begin{aligned}
& \left|-\log k \int_{0}^{\infty} r e^{-k r} \int_{0}^{r} r^{\prime} d \mu\left(r^{\prime}\right) d \mu(r)+\log k \int_{0}^{\infty} r \int_{0}^{r} r^{\prime} d \mu\left(r^{\prime}\right) d \mu(r)\right| \\
& \quad \leqq(-\log k) c_{2} \int_{0}^{\infty} r \frac{k r}{1+k r} \int_{0}^{r} r^{\prime} d \mu\left(r^{\prime}\right) d \mu(r)\left(\text { because } 1-e^{-k r} \leqq c_{2} \frac{k r}{1+k r}\right) \\
& \quad \leqq c_{2}|\log k| \int_{0}^{\infty} \frac{k r^{2}}{1+k r} d \mu(r) \int_{0}^{\infty} r^{\prime} d \mu\left(r^{\prime}\right) \rightarrow 0 . \quad(k \rightarrow 0)
\end{aligned}
$$

The second term evidently approaches to

$$
-\int_{0}^{\infty} r(\gamma+\log (r / 2)) \int_{0}^{r} r^{\prime} d \mu\left(r^{\prime}\right) d \mu(r)
$$

as $k \rightarrow 0$ and the last term tends to zero. Combining these results we obtain the last inequality in $(6.6)^{\prime}$.

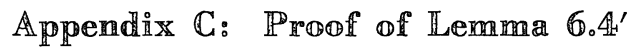

By the same argument as in the proof of Lemma 6.4, it is sufficient to prove

$$
\left\|g_{2}^{0}-g_{2}^{0}(k)\right\|_{2}^{2}=o\left(|\log k|^{-1}\right), \quad(k \rightarrow 0)
$$


the left hand side of which is given, from $(6.5)^{\prime}$ and $(6.5)_{0}^{\prime}$, as

$$
\frac{2}{(\log k)^{2}} \int_{0}^{\infty} r \int_{0}^{r} r^{\prime}\left(K_{0}(k r) I_{0}\left(k r^{\prime}\right)+\log k\right)^{2} d \mu\left(r^{\prime}\right) d \mu(r) .
$$

The behaviours of $I_{0}$ and $K_{0}$ given in Appendix A imply

$$
\left|K_{0}(x) I_{0}\left(x^{\prime}\right)+\log x\right| \leqq b_{1} \text {, for } 0 \leqq x^{\prime} \leqq x \leqq 1 \text { ( } b_{1} \text { : constant) }
$$

and

$$
\left|K_{0}(x) I_{0}\left(x^{\prime}\right)\right| \leqq b_{2}, \quad \text { for } x \geqq 1 \text { and } x \geqq x^{\prime} \geqq 0 \text { ( } b_{2} \text { : constant). }
$$

\section{Consequently}

$$
\begin{aligned}
& \frac{1}{(\log k)^{2}} \int_{0}^{1} r \int_{0}^{r} r^{\prime}\left(K_{0}(k r) I_{0}\left(k r^{\prime}\right)+\log k\right)^{2} d \mu\left(r^{\prime}\right) d \mu(r) \\
& \leqq \frac{1}{(\log k)^{2}} \int_{0}^{1} r\left(|\log r|+b_{1}\right)^{2} \int_{0}^{r} r^{\prime} d \mu\left(r^{\prime}\right) d \mu(r) \\
& \leqq \frac{1}{(\log k)^{2}} \int_{0}^{1} r\left(|\log r|+b_{1}\right) \int_{0}^{r} r^{\prime}\left(\left|\log r^{\prime}\right|+b_{1}\right) d \mu\left(r^{\prime}\right) d \mu(r) \\
& =O\left((\log k)^{-2}\right), \quad(k \rightarrow 0)
\end{aligned}
$$

and

$$
\begin{aligned}
& \frac{1}{(\log k)^{2}} \int_{1 / k}^{\infty} r \int_{0}^{r} r^{\prime}\left(K_{0}(k r) I_{0}\left(k r^{\prime}\right)+\log k\right)^{2} d \mu\left(r^{\prime}\right) d \mu(r) \\
& =\frac{\left(b_{2}+|\log k|\right)^{2}}{(\log k)^{2}} \int_{1 / k}^{\infty} r \int_{0}^{r} r^{\prime} d \mu\left(r^{\prime}\right) d \mu(r) \\
& =\frac{\left(b_{2}+|\log k|\right)^{2}}{|\log k|^{3}} \int_{1 / k}^{\infty} r|\log r| d \mu(r) \int_{0}^{\infty} r^{\prime} d \mu\left(r^{\prime}\right)=o\left(|\log k|^{-1}\right) .
\end{aligned}
$$

As for the integral

$$
\begin{gathered}
\frac{1}{(\log k)^{2}} \int_{1}^{1 / k} r \int_{0}^{r} r^{\prime}\left(K_{0}(k r) I_{0}(k r)+\log k\right)^{2} d \mu\left(r^{\prime}\right) d \mu(r) \\
=\frac{1}{|\log k|}\left\{\int_{1}^{1 / k} r \int_{0}^{r} r^{\prime}\left|K_{0}(k r) I_{0}\left(k r^{\prime}\right)+\log k\right|\right. \\
\left.\frac{\left|K_{0}(k r) I_{0}\left(k r^{\prime}\right)+\log k\right|}{|\log k|} d \mu\left(r^{\prime}\right) d \mu(r)\right\},
\end{gathered}
$$


we have the inequalities

$$
\left|K_{0}(k r) I_{0}\left(k r^{\prime}\right)+\log k\right| \leqq|\log r|+b_{1}
$$

and

$$
\frac{\left|K_{0}(k r) I_{0}\left(k r^{\prime}\right)+\log k\right|}{|\log k|} \leqq \frac{|\log r|+b_{1}}{|\log k|} \leqq \frac{|\log k|+b_{1}}{|\log k|}
$$

both of which hold in the range of integration. Since the integrand tends to zero as $k \rightarrow 0$, the expression in the curly brackets tends to zero.

\section{Appendix D: Estimate on $\left(\tilde{\mathcal{F}} G_{2}^{0}(k) \tilde{P}\right)\left(r, r^{\prime}\right)$}

The positivity of $\dot{P} G_{2}^{0}(k) \tilde{P}$ implies

$$
\left(\tilde{P} G_{2}^{0}(k) \tilde{P}\right)\left(r, r^{\prime}\right)^{2} \leqq\left(\tilde{P} G_{2}^{0}(k) \tilde{P}\right)(r, r) \cdot\left(\tilde{P} G_{2}^{0}(k) \tilde{P}\right)\left(r^{\prime}, r^{\prime}\right),
$$

where

$$
\begin{aligned}
& \left(\tilde{P} G_{2}^{0}(k) \tilde{P}\right)(r, r)=r\left\{I_{0}(k r) K_{0}(k r)-2 \tilde{\tau}_{0} K_{0}(k r) \int_{0}^{r} s I_{0}(k s) d \mu(s)\right. \\
& \left.-2 \tilde{\tau}_{0} I_{0}(k r) \int_{r}^{\infty} s K_{0}(k s) d \mu(s)+2 \tilde{\tau}_{0}^{2} \int_{0}^{\infty} s K_{0}(k s)\left\{\int_{0}^{s} t I_{0}(k t) d \mu(t)\right\} d \mu(s)\right\} .
\end{aligned}
$$

By virtue of the properties of $I_{0}$ and $K_{0}$ given in Appendix A, we obtain the following estimate:

$$
\left(\tilde{P} G_{2}^{0}(k) \tilde{P}\right)(r, r) \leqq F_{1}(r ; k)+F_{2}(r),
$$

where $F_{1}$ is equal to

$$
\begin{gathered}
(-\log k) r\left\{1-2 \tilde{\tau}_{0} \int_{0}^{\infty} s e^{-k|r-s|} d \mu(s)\right. \\
\left.\quad+\tilde{\tau}_{0}^{2} \int_{0}^{\infty} s\left\{\int_{0}^{\infty} t e^{-k|s-t|} d \mu(t)\right\} d \mu(s)\right\} \\
=(-\log k) r\left\{2 \tilde{\tau}_{0} \int_{0}^{\infty} s\left(1-e^{-k|r-s|}\right) d \mu(s)\right. \\
\left.\quad-\tilde{\tau}_{0}^{2} \int_{0}^{\infty} s\left\{\int_{0}^{\infty} t\left(1-e^{-k|s-t|}\right) d \mu(t)\right\} d \mu(s)\right\},
\end{gathered}
$$


and $F_{2}$ is a positive function independent of $k$ such that

$$
\int_{0}^{\infty} F_{2}(r) d \mu(r)<\infty
$$

From the inequalities

$$
1-e^{-k|r-s|} \leqq c_{2} \frac{k|r-s|}{1+k|r-s|} \leqq c_{2}\left[\frac{k r}{1+k r}+\frac{k s}{1+k s}\right]
$$

we obtain

$$
\begin{gathered}
\int_{0}^{\infty} F_{1}(r ; k) d \mu(r)=\tilde{\tau}_{0}(-\log k) \int_{0}^{\infty} r\left\{\int_{0}^{\infty} s\left(1-e^{-k|r-s|}\right) d \mu(s)\right\} d \mu(r) \\
\leqq 2 c_{2} \tilde{\tau}_{0}(-\log k) \int_{0}^{\infty} \frac{k r^{2}}{1+k r} d \mu(r),
\end{gathered}
$$

which tends to zero as $k \rightarrow 0$ by (A.3). Consequently the integral

$$
\left\{\left(\int_{0}^{N^{-1}}+\int_{N}^{\infty}\right) d \mu(r)\left(\int_{0}^{N^{-1}}+\int_{N}^{\infty}\right) d \mu\left(r^{\prime}\right)\right\} \cdot\left(P G_{2}^{0}(k) \tilde{P}\right)\left(r, r^{\prime}\right)^{2}
$$

tends to zero if we let $k \rightarrow 0$ and $N \rightarrow \infty$ independently.

\section{References}

[1] Bargmann, V., On the number of bound states in a central field of force, Proc. Nat. Acad. Sci. U.S. A. 38 (1952), 961-966.

[2] Schwinger, J., On the bound states of a given potential, Proc. Nat. Acad. Sci. U.S.A. 47 (1960), 122-129.

[3] Newton, R. G., Nonrelativistic S-matrix poles for complex angular momenta, Jour. Math. Phys. 3 (1962), 867-882.

1 41 See, for instance, Vilenkin, N. Ya., Sperial Functions and the Theory of Group Representations Chap. IX, $\$ 2.5$ (Moscow, 1965).

L5 Kodaira, K., The eigenvalue problem for ordinary differential equations of the second order and Heisenberg's theory of S-inatrices, Amcr. Jour. Math. 71 (1949), 921-944.

[6] De Alfaro, V. and Regge, T., Potential Srattering, Chaps. 3 and 4 (North-Holland, 1965).

[7] Riesz, F. and Sz.-Nagy, B., Functional Analysis, Chap. IX, §136 (English transl. Frederic Ungar, 1953).

[8] ibid. Chap. VI, $\$ 98$.

[9] ibid. Chap. VI, \$95. 
